\author{
W. Duke
}

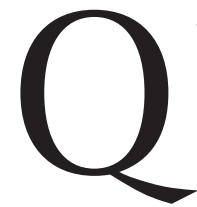

uite a few famous and extraordinarily gifted mathematicians led lives that were tragically cut short. Ramanujan is certainly among them. While suffering from a fatal disease, he discovered what he called mock theta functions. Three months before his death in 1920 at the age of thirty-two, he described them in a letter to Hardy that was written under difficulties and is in places very obscure [30, p. 354]. One fascinating and also frustrating aspect of this story is that from the start the precise definition of a mock theta function has been elusive. Ramanujan did not give a formal definition but explained what properties a mock theta function should have and illustrated them through several examples. He also gave some of their properties without proof. Subsequent work stimulated by his letter (and his "lost" notebook discovered later) has been of two somewhat different types. The goal of the first type, beginning with Watson's cleverly titled address "The Final Problem...", has been to prove Ramanujan's claims on his terms and to give extensions of the same nature. Here the focus is not so much on the definition but rather on the examples. The mathematics is intricate and beautiful but leaves unclear a broader theoretical role for mock theta functions.

The second type, which is more recent, has clarified this role. We now understand that mock theta functions can be regarded as incomplete harmonic Maass forms and thus belong naturally to the theory of modular forms. This has led to new results about mock theta functions and,

W. Duke is professor of mathematics at UCLA. His email address iswdduke@ucla.edu.

DOI: http://dx.doi.org/10.1090/noti1185 perhaps more interestingly, to a resurgence in the study of other kinds of harmonic Maass forms and their arithmetic properties. Mock theta functions are now seen as interesting examples of a much larger class of mock modular forms. These have applications to elliptic curves, singular moduli and their real quadratic analogues, Borcherds products, Eichler cohomology, and Galois representations, among others.

My main goal is to give a reasonably short (hence necessarily incomplete) introduction to this story that is accessible to a nonspecialist. At the same time I will occasionally make use of terms I have not defined and provide technical details for the benefit of those having more extensive background knowledge. A number of excellent expositions and survey articles have appeared on mock theta functions and their generalizations: [1], [9], [17], [18], [27], [28], [37]. In particular, this paper has quite a lot in common with Folsom's 2010 article [17] in the Notices. Here I give a complementary account along somewhat similar lines but with more detail and also outline further developments. Any serious discussion of Ramanujan's mathematics must contain identities between complex formulas. However, there are also conceptual aspects, and my aim is to give a treatment that illuminates both.

\section{Ramanujan's Letter}

The mathematical part of Ramanujan's letter is reproduced in [1]. Ramanujan motivated his mock theta functions by first describing briefly two "genuine" theta functions. The first example is the $q$-series for the partition function $p(n)$ :

$$
g(q)=\sum_{n \geq 0} p(n) q^{n}=\prod_{m \geq 1}\left(1-q^{m}\right)^{-1} .
$$


A $q$-series is simply a power series in powers of $q$ whose coefficients usually count something of combinatorial interest. Here $p(n)$ counts the number of ways of expressing $n$ as a sum of positive integers, repetition allowed and not counting order. For example, $p(6)=11$, since

$$
\begin{aligned}
6 & =5+1=4+2=4+1+1=3+3 \\
& =3+2+1=3+1+1+1=2+2+2 \\
& =2+2+1+1=2+1+1+1+1 \\
& =1+1+1+1+1+1 .
\end{aligned}
$$

Ramanujan's second example of a genuine theta function is the $q$-series

(3)

$$
h(q)=\sum_{n \geq 0} \hat{p}(n) q^{n}=\prod_{m \geq 1} \frac{1}{\left(1-q^{5 m-1}\right)\left(1-q^{5 m-4}\right)},
$$

where now $\hat{p}(n)$ equals the number of partitions into parts of the forms $5 m-1$ and $5 m-4$.

A mock theta function, as conceived by Ramanujan, is a special kind of $q$-series that mimics two properties of $g(q)$ and $h(q)$. The first is that they can be expressed in Eulerian form:

$$
g(q)=1+\sum_{n \geq 1} \frac{q^{n^{2}}}{(1-q)^{2}\left(1-q^{2}\right)^{2} \cdots\left(1-q^{n}\right)^{2}},
$$

$$
h(q)=1+\sum_{n \geq 1} \frac{q^{n^{2}}}{(1-q)\left(1-q^{2}\right) \cdots\left(1-q^{n}\right)} .
$$

Here "Eulerian form" refers to the basic shape of the series in (4) and (5); such series have coefficients that count restricted partitions of various types. For example, (5) is one of the Rogers-Ramanujan identities and expresses the fact that $\hat{p}(n)$ equals the number of partitions of $n$ into parts having minimal difference two.

The second property is that the $q$-series converge for $|q|<1$ and have the unit circle as a natural boundary but are amenable to the circle method. ${ }^{1}$ The circle method was actually invented by Hardy and Ramanujan in one of their most important collaborations [19] to obtain a remarkably accurate formula for $p(n)$. Their idea was to apply Cauchy's integral formula to $g(q)$.

As a model example, consider the number of partitions $p_{3}(n)$ of $n$ into parts $\leq 3$. For instance, it is easily checked from (2) that $p_{3}(6)=7$. Now $p_{3}(n)$ has the generating function

$$
g_{3}(q)=\sum_{n \geq 0} p_{3}(n) q^{n}=\frac{1}{(1-q)\left(1-q^{2}\right)\left(1-q^{3}\right)},
$$

and Cauchy's formula gives for $c<1$ that

$$
p_{3}(n)=\frac{1}{2 \pi i} \int_{|q|=c} q^{-n-1} g_{3}(q) d q \text {. }
$$

\footnotetext{
${ }^{1}$ This is not exactly how Ramanujan put it, but it captures the idea.
}

Next we push the contour over the singularities of $g$. By letting $c \rightarrow \infty$ we pick up residues from the poles to get the exact formula

(6) $p_{3}(n)=\frac{1}{12}(n+3)^{2}+\left(\frac{1}{2} \cos \frac{1}{2} \pi n\right)^{2}-\left(\frac{2}{3} \sin \frac{1}{3} \pi n\right)^{2}$.

It follows that $p_{3}(n)$ is the integer nearest to $\frac{1}{12}(n+3)^{2}$.

The function $g(q)$ in (1) is much harder to use in this way, since it has the unit circle as a natural boundary but near roots of unity it can be well approximated. Thus Hardy and Ramanujan could not simply push the contour over the singularities but had the brilliant idea of moving it toward the unit circle in a cunning way (the circle method) and approximating the influence of the singularities. This process is greatly facilitated by modular properties of $g(q)$, which yield a precise approximation of $g(q)$ near each root of unity. The most influential singularity is at $q=1$ and for this we use the modular inversion formula

$$
q^{1 / 24} g(q)=\sqrt{y}\left(q^{\prime}\right)^{1 / 24} g\left(q^{\prime}\right),
$$

when $q=e^{-2 \pi y}$ and $q^{\prime}=e^{-\frac{2 \pi}{y}}$. Now (7) easily gives

$$
\sqrt{y} \exp \frac{\pi}{12}\left(\frac{1}{y}-y\right)
$$

as the first-order approximation to $g\left(e^{-2 \pi y}\right)$ as $y \rightarrow 0$. This leads to the asymptotic formula

$$
p(n) \sim \frac{1}{4 n \sqrt{3}} \exp \left(\pi \sqrt{\frac{2 n}{3}}\right)
$$

as $n \rightarrow \infty$. Making full use of the modular properties of $g(q)$, Hardy and Ramanujan extended this idea to obtain an infinite sum with the property that $p(n)$ is the integer closest to the sum of the first $\sqrt{n}$ terms for sufficiently large $n$. Rademacher [30] (and independently Selberg) later refined their method to get a variation that is exact:

(10)

$$
p(n)=\frac{1}{\sqrt{6} \pi} \sum_{k \geq 1} k S_{k}(n) \frac{d}{d n}\left(\lambda(n)^{-1} \sinh \left(\frac{\sqrt{2} \pi \lambda(n)}{\sqrt{3} k}\right)\right)
$$

where $\lambda(n)=\left(n-\frac{1}{24}\right)^{1 / 2}$. Also, after Selberg,

$$
S_{k}(n)=\sum(-1)^{\ell} \cos \frac{6 \ell-1}{6 k} \pi,
$$

the sum being over $\ell$ modulo $2 k$ with $\frac{3 \ell^{2}-\ell}{2} \equiv-n$ $(\bmod k)$. In a sense, (10) is like (6) only much more difficult to derive. Selberg gives in [33] an interesting account of (10) and speculates about why Hardy and Ramanujan didn't give this refinement themselves. In any case, the fact that a purely arithmetic counting function can be computed by such a formula is astonishing and must have motivated Ramanujan to look for similar $q$-series. In particular, the function $h(q)$ also has modular properties that yield good approximations at roots of unity and is amenable to the circle method. 
Ramanujan's first example of a mock theta function is the Eulerian series

(12)

$$
\begin{aligned}
f(q) & =1+\sum_{n \geq 1} a(n) q^{n} \\
& =1+\sum_{n \geq 1} \frac{q^{n^{2}}}{(1+q)^{2}\left(1+q^{2}\right)^{2} \cdots\left(1+q^{n}\right)^{2}},
\end{aligned}
$$

which appears deceptively similar to $g(q)$ and $h(q)$. The coefficient $a(n)$ counts the number of partitions of $n$ with even rank minus the number with odd rank, where the rank of a partition is its largest part minus the number of its parts. Ramanujan gives information about the behavior of $f(q)$ near each root of unity that is similar yet also different enough from that for genuine theta functions that he concludes that it is inconceivable that a single 9 -function could be found to cut out the singularities of $f(q)$. Nevertheless, he was able to give an asymptotic formula for the coefficient $a(n)$ that comes from the circle method and whose leading term is given by

$$
a(n) \sim \frac{(-1)^{n-1}}{2 \sqrt{n-\frac{1}{24}}} \exp \left(\pi \sqrt{\frac{n}{6}-\frac{1}{144}}\right)
$$

as $n \rightarrow \infty$.

Ramanujan gave some seventeen examples of mock theta functions and stated for them numerous identities and asymptotic properties. Andrews found more examples in Ramanujan's "lost" notebook, which he argues was written near the end of his life. Andrews [1], Gordon [18], Selberg [31] and Watson [35], among others, proved Ramanujan's claims and greatly expanded their extent, giving transformation formulas connecting them and combinatorial interpretations of their coefficients. Of particular interest are identities for certain "fifth order" mock theta functions found in the lost notebook, called by Andrews and Garvan [2] the Mock Theta Conjectures. The proof of these and others for mock theta functions of seventh order were given by Hickerson [21], [22]. See [1] for a wide-ranging survey and references on such problems (i.e., of the first type) about mock theta functions.

Ramanujan emphasized that a mock theta function should not have the same singularities at roots of unity as a genuine theta function. Exactly what he meant by a genuine theta function was never made clear, although various plausible explanations have been given. It seems to me that this issue is a bit of a red herring. After all, the really interesting problem is to explain structurally what unites these examples. In other words, it is more fruitful to regard $f(q)$ as a generalization of $g(q)$ and $h(q)$ and determine how it generalizes them.

For example, one of Ramanujan's results from the lost notebook (see [1, p. 287]), later rediscovered by Watson [35], is that $f(q)$ is related under a modular transformation to another mock theta function:

$$
\omega(q)=\sum_{n \geq 0} \frac{q^{2 n(n+1)}}{(1-q)^{2}\left(1-q^{3}\right)^{2} \cdots\left(1-q^{2 n+1}\right)^{2}} .
$$

Thus if $q=e^{-2 \pi y}$ and $q_{1}=e^{-\frac{\pi}{2 y}}$, then

$$
\begin{gathered}
q^{-1 / 24} f(q)=2 y^{-1 / 2} q_{1}^{4 / 3} \omega\left(q_{1}^{2}\right)+4(3 y)^{1 / 2} \\
\quad \times \int_{0}^{\infty} e^{-3 \pi y x^{2}} \frac{\sinh 2 \pi y x}{\sinh 3 \pi y x} d x .
\end{gathered}
$$

This suggestive formula hints that the mock theta function $f(q)$ secretly wants to be modular.

\section{Mock Theta Functions Today}

Modular forms can be defined in many different ways depending on one's point of view. For our purposes here they are smooth complex-valued functions $F$ defined on the upper half-plane $\mathcal{H}$ that transform in a certain way under a finite-index subgroup $\Gamma$ of $\operatorname{SL}(2, \mathbb{Z})$, where $\gamma \in \Gamma$ acts on $\mathcal{H}$ by linear fractional maps $z \mapsto \gamma z=\frac{a z+b}{c z+d}$. We say that $F$ has weight $k \in \mathbb{Q}$ for $\Gamma$, if for all $\gamma=\left(\begin{array}{ll}a & b \\ c & d\end{array}\right) \in \Gamma$,

$$
F(\gamma z)=\chi(\gamma)(c z+d)^{k} F(z),
$$

where $|\chi(\gamma)|=1$ depends only on $\gamma$ and the branch of $\log$ chosen to define $(c z+d)^{k}$. In addition, we suppose that $F(z)$ satisfies a growth condition, conveniently given by requiring that the invariant function $\operatorname{Im}(z)^{k / 2}|F(z)|$ is bounded in $\mathcal{H}$ by a constant times $\exp \left(C\left(\operatorname{Im}(z)+\operatorname{Im}(z)^{-1}\right)\right)$ for some constant $C \geq 0$.

The generating function for the partition function gives rise to a modular form,

$$
G(z)=q^{1 / 24} g(q)=q^{-1 / 24} \prod_{m \geq 1}\left(1-q^{m}\right)^{-1},
$$

where we set for $z \in \mathcal{H}$,

$$
q=e(z)=\exp (2 \pi i z)
$$

Dedekind showed that $G(z)^{-1}=\eta(z)$ has weight $1 / 2$ for $\operatorname{SL}(2, \mathbb{Z})$, this being Dedekind's famous eta function, so $G(z)$ has weight $-1 / 2$ for $\operatorname{SL}(2, \mathbb{Z})$. The behavior of $g$ near roots of unity is determined by that of $G(z)$ near rational points, and this can be deduced from the transformation formula. In particular, (7) follows from the transformation formula with $\gamma z=-1 / z$. The Rademacher formula uses explicit knowledge of the $\chi(\gamma)$ in the transformation formula for all $\gamma \in \operatorname{SL}(2, \mathbb{Z})$, which was found by Dedekind in terms of the Dedekind sum and is a $24^{\text {th }}$ root of unity.

Similarly, the Rogers-Ramanujan function

$$
H(z)=q^{-1 / 60} \prod_{m \geq 1}\left(1-q^{5 m-1}\right)^{-1}\left(1-q^{5 m-4}\right)^{-1}
$$

is a modular form of weight 0 for $\Gamma(5)$. Here $\Gamma(N)$, the principal congruence subgroup of level $N \in \mathbb{Z}^{+}$, comprises $\gamma \in \operatorname{SL}(2, \mathbb{Z})$ with $\gamma \equiv\left(\begin{array}{ll}1 & 0 \\ 0 & 1\end{array}\right)(\bmod N)$. 
The functions $G$ and $H$ are holomorphic in $\mathcal{H}$ and are related to the $g$ and $h$ of (4) and (5) simply by multiplication by a fractional power of $q$. In order to give the modular form definition of mock theta functions, we must consider Maass forms. There is a weight $k$ version of the Laplace operator defined through

$$
\Delta_{k} F=-y^{2}\left(\partial_{x}^{2}+\partial_{y}^{2}\right)+i k y\left(\partial_{x}+i \partial_{y}\right)
$$

If $F$ has weight $k$ for $\Gamma$, then so does $\Delta_{k} F$. This follows most easily from the decomposition $\Delta_{k}=\xi_{2-k} \circ \xi_{k}$, where

$$
\xi_{k} F(z)=2 i y^{k} \overline{\partial_{\bar{z}} F(z)},
$$

since it is easily checked that $\xi_{k} F$ has weight $2-k$ when $F$ has weight $k$.

A Maass form of weight $k$ for $\Gamma$ is a modular form $F$ of weight $k$ for $\Gamma$ that is an eigenfunction of $\Delta_{k}$ :

$$
\Delta_{k} F=\lambda F .
$$

In case $\lambda=0$ we say that $F$ is harmonic of weight $k$. If $F$ is holomorphic it is also harmonic of weight $k$ for any $k$ by (16), so the class of harmonic Maass forms of weight $k$ includes the holomorphic forms.

Around 2000 Zwegers [39], [40] made a breakthrough in our understanding of Ramanujan's mock theta functions. It follows from his work that, after multiplication by a power of $q$ and the addition of a certain nonholomorphic function, a mock theta function is a harmonic Maass form of weight $1 / 2$. For the example $f(q)$ from (12),

$$
F(z)=q^{-\frac{1}{24}} f(q)+g^{*}(z)
$$

is a harmonic Maass form of weight $1 / 2$ for $\Gamma(2)$ when

$$
g^{*}(z)=\sum_{n \in 1+6 \mathbb{Z}} \operatorname{sgn}(n) \beta\left(\frac{n^{2} y}{6}\right) q^{-n^{2} / 24} .
$$

Here $\beta(x)$ is defined for $x>0$ in terms of the complementary error function and the standard incomplete gamma function by

$$
\begin{aligned}
& \beta(x)=\operatorname{erfc}(\sqrt{\pi x})=\frac{1}{\sqrt{\pi}} \Gamma\left(\frac{1}{2}, \pi x\right), \\
& \text { where } \Gamma(s, x)=\int_{x}^{\infty} t^{s} e^{-t} \frac{d t}{t} .
\end{aligned}
$$

Now $g^{*}(z)$ satisfies the equation

$$
\xi_{1 / 2} g^{*}(z)=\frac{\sqrt{6}}{3} \sum_{n \in 1+6 \mathbb{Z}} n q^{n^{2} / 24},
$$

and the right-hand side of (19) is a unary theta function (a cusp form) of weight $3 / 2$, called by Zagier a shadow of $f$. An explicit determination of the transformation formula of $F$ allowed Bringmann and Ono [5] to prove an exact formula of Rademachertype for the coefficients of Ramanujan's mock theta function $f(q)$. This formula, which refines Ramanujan's first-order asymptotic (13), had been conjectured by Andrews and Dragonette (see [5] for references). Combined with Rademacher's formula it gives exact formulas for the number of partitions with even (resp. odd) ranks.

This is but one of numerous recent results about mock theta functions stimulated, at least in part, by the work of Zwegers. See [27] and [37] for a lot more. In fact, a connection between Maass forms and mock theta functions was already found in 1988 by Cohen [10], but his results are much more closely connected with the theory of quantum modular forms [38] than with the main theme of this paper.

\section{Modular Forms as $q$-series and $L$-functions}

Today we understand that mock theta functions provide but one class of examples of harmonic Maass forms. Before discussing some others, I will give a whirlwind overview of some aspects of the theory of modular forms as $q$-series and their associated $L$-functions. This should help to place these examples in a broader context. Standard references for this section are the books [24] and [25]. An older but still valuable article on the Fourier coefficients of modular forms is [32]. The reader may also consult [26] by Iwaniec and Sarnak for an extensive overview of further developments.

Modular forms as $q$-series really began with Jacobi and his theta functions. Thus for $m \in \mathbb{Z}^{+}$ the function

$$
\vartheta(z)^{m}=\left(\sum_{n \in \mathbb{Z}} q^{n^{2}}\right)^{m}=\sum_{n \geq 0} r_{m}(n) q^{n}
$$

is a modular form of weight $m / 2$, where $r_{m}(n)$ is the number of representations of $n$ as the sum of $m$ squares. Ramanujan took the connection to another level, remarkably through an example known to Jacobi, namely,

(21)

$$
\Delta(z)=\eta(z)^{24}=q \prod_{m \geq 1}\left(1-q^{m}\right)^{24}=\sum_{n \geq 1} \tau(n) q^{n},
$$

which is a modular form of weight 12 for $\operatorname{SL}(2, \mathbb{Z})$. This modular form is a cusp form, which in this situation means simply that the constant term in the $q$-series (21) vanishes. Alternatively, $\Delta(z)$ being a cusp form is equivalent to having $\operatorname{Im}(z)^{6}|\Delta(z)|$ be bounded on $\mathcal{H}$. Ramanujan made two conjectures about the Fourier coefficients $\tau(n)$, which are obviously integers. Both had a profound influence on the development of the theory of modular forms.

The first conjecture implies that $\tau(n)$ is a multiplicative arithmetic function and can be succinctly stated by saying that its associated $L$-series has an Euler product:

$$
L(s)=\sum_{n \geq 1} \tau(n) n^{-s}=\prod_{p}\left(1-\tau(p) p^{-s}+p^{11-2 s}\right)^{-1},
$$


where $p$ runs over the primes. This was soon proved by Mordell and served as the starting point for Hecke's theory of Hecke operators and $L$-functions associated to holomorphic cusp forms. In this example $L(s)$ is entire in $s$ and satisfies a functional equation like that of the Riemann zeta function.

The second conjecture, known as the Ramanujan conjecture, states that for a prime $p$,

$$
|\tau(p)| \leq 2 p^{\frac{11}{2}} .
$$

As Hardy showed, Cauchy's formula immediately gives the bound $|\tau(n)| \ll n^{6}$. Note that $\tau(n)$ is small in comparison with the size of the partition function $p(n)$, whose asymptotic formula was given in (9). This is explained by the fact that the asymptotic formula for $p(n)$ gets exponential terms from the singularities of its generating function $G(z)$, which was given in (15). The first nontrivial assault on (22) was made by Kloosterman, who applied the circle method and reduced the estimation of $\tau(n)$ (and the coefficients of other cusp forms) to that of Kloosterman sums. In their simplest form these are the exponential sums

$$
K(n, m ; c)=\sum e\left(\frac{n x+m x^{-1}}{c}\right)
$$

where $c>0$ and the sum is over $x(\bmod c)$ with $(x, c)=1$ and $x^{-1} x \equiv 1(\bmod c)$. Such sums show up in the circle method in a way similar to that of the sums $S_{k}(n)$ in (11) in the Rademacher/Selberg formula for $p(n)$. Weil's sharp estimate for Kloosterman sums, a consequence of the Riemann hypothesis for curves, yielded the exponent $23 / 4+\epsilon$ in (22). The full conjecture is deeper and was finally established by Deligne.

Maass extended Hecke's theory to Maass cusp forms and their $L$-functions. Selberg developed the spectral theory of Maass forms and gave applications through his trace formula. Consideration of nonholomorphic forms was an essential generalization that opened the way for both specific applications and broad generalizations to automorphic representations of Lie groups and also, through their associated $L$-functions, to the Langlands program. One of the many motivations behind the Langlands program is to prove the analogue of the Ramanujan conjecture for the coefficients of a Maass cusp form, which is still open. Supporting data was computed to great accuracy by Hejhal [20] in the 1980s (see [3] for recent work). For example, numerically the smallest eigenvalue $\lambda$ from (17) of a nonzero even Maass cusp form $F$ of weight $k=0$ for $\operatorname{SL}(2, \mathbb{Z})$ is $\lambda=190.13154 \ldots$, and the coefficients of its associated $L$-function

$$
\begin{aligned}
L(s) & =\prod_{p}\left(1-\alpha(p) p^{-s}+p^{-2 s}\right)^{-1} \\
& =1+(1.54930 \ldots) 2^{-s}+(0.24689 \ldots) 3^{-s}+\cdots
\end{aligned}
$$

are conjectured to satisfy $|a(p)| \leq 2$ for all primes $p$. In general, the coefficients of Maass forms are quite mysterious and present many unsolved problems.

\section{Harmonic Maass Forms}

It is especially interesting therefore that mock theta functions can be completed to become harmonic Maass forms. Of course harmonic Maass forms are quite special (with eigenvalue $\lambda=0$ ), and this is one reason behind their having more accessible arithmetic properties. It is sometimes convenient to use terminology due to Zagier and call a $q$-series a mock modular form of weight $k$ if it can be completed as before to a harmonic Maass form $F$. Such a mock modular form has as a shadow a nonzero constant multiple of $\xi_{k} F$, which is a holomorphic form of weight $2-k$. Roughly speaking, a harmonic Maass form of weight $k$ has a "holomorphic part," which is a mock modular form, and a shadow (normalized in some way), which is a holomorphic form of weight $2-k$.

An early example of a mock modular form of weight $3 / 2$ was found in [23] and is given by the Eisenstein series

$$
\begin{aligned}
E_{3 / 2}(z) & =-\frac{1}{12}+\sum_{n \geq 1} H(n) q^{n} \\
& =-\frac{1}{12}+\frac{1}{3} q^{3}+\frac{1}{2} q^{4}+q^{7}+\cdots,
\end{aligned}
$$

where $H(n)$ are the Hurwitz class numbers, which count (with weights) the number of classes of binary quadratic forms of discriminant $-n$. Although it was not stated in this form, it was shown there that the Jacobi theta function $9(z)$ from $(20)$ is a shadow of $E_{3 / 2}(Z)$.

A natural problem is to try to understand the coefficients of a mock modular form having as a shadow a given holomorphic modular form. For example, Bruinier and Ono [7] applied this idea to cusp forms of weight $3 / 2$ whose Shimura lifts are cusp forms of weight 2 associated to certain elliptic curves. They found mock modular forms of weight $1 / 2$ that are not classical mock theta functions but whose coefficients contain information about the $L$-series of the curves. Related to this are interesting questions about the algebraicity of the coefficients of harmonic Maass forms (see [29], [8], and their references).

Another catalyst for recent interest in harmonic Maass forms was the work of Borcherds on monstrous moonshine, which as a byproduct created new interest in modular forms with singularities (see e.g., [4], [6]). In particular, this has led to various applications and generalizations of the Borcherds product (see [28] for some further references). In [12], mock modular forms of weight $1 / 2$ for $\operatorname{SL}(2, \mathbb{Z})$ are constructed whose coefficients are cycle integrals of the modular $j$-function. They 
have as shadows certain meromorphic modular forms of weight $3 / 2$ studied by Zagier [36] with coefficients that are traces of singular moduli. Associated to these mock modular forms are real quadratic analogues of Borcherds products. In addition, a real quadratic analogue of (23) is constructed. See also [11], [13].

There are interesting examples of mock modular forms in integral weights as well. An example is the Eisenstein series

$$
\begin{aligned}
E_{2}(z) & =1-24 \sum_{n \geq 1} \sigma(n) q^{n} \\
& =1-24 q-72 q^{2}-96 q^{3}-\cdots,
\end{aligned}
$$

where $\sigma(n)=\sum_{d \mid n} d$ is the sum of divisors function. It follows from an early result of Hecke that $E_{2}(z)$ is mock modular of weight 2 for the full modular group with the constant function 1 as a shadow. Other mock modular forms of weight 2 whose shadows are weakly holomorphic modular functions are studied in [14]. Another kind of example is given by an Eichler integral of a holomorphic modular form of weight $k \in 2 \mathbb{Z}^{+}$, which may be considered to be mock modular of weight $2-k$ (see [37]).

Mock modular forms in the self-dual case of weight 1 with coefficients that can be expressed in terms of logs of algebraic numbers from certain Hilbert class fields are studied in [15], [16], and [34]. These forms have shadows whose $L$-functions are Artin $L$-functions associated to dihedral Galois representations. This phenomenon appears, at least numerically, to hold for exotic representations as well [15]. It is closely connected with Stark's conjectures on special values of derivatives of $L$-functions.

Finally, it should be noted that harmonic Maass forms with singularities do not respect Hecke operators like those without singularities. Therefore they do not fit directly into the usual automorphic representation picture. It is possible that a shadow of such a form is a Hecke eigenform and hence has an associated $L$-function. This gives an indirect connection, but one that is still not very well understood in general. While we now have a better understanding of what a mock theta function is and have examples of other kinds of harmonic Maass forms with arithmetic properties, we are still far from an answer to the question: what broader role do general harmonic Maass forms play in arithmetic?

\section{Acknowledgments}

I thank Bill Casselman for some excellent suggestions improving an early version of this paper. I also thank Scott Ahlgren, Özlem Imamoḡlu, Peter Sarnak, and the referees for their comments.

\section{References}

1. GEORge E. ANDrews, Mock theta functions, Theta functions, 1987, Part 2 , 283-298, Proc. Sympos. Pure Math., 49, Part 2, Amer. Math. Soc., Providence, RI, 1989.

2. GEORGE E. ANDREWS and F. G. GARVAN, Ramanujan's "lost" notebook. VI. The mock theta conjectures, $A d v$. in Math. 73 (1989), no. 2, 242-255.

3. ANDREW R. BOOKER, ANDREAS STRÖMBERGSSON, and AKSHAY VENKATESH, Effective computation of Maass cusp forms, Int. Math. Res. Not. 2006, Art. ID 71281, $34 \mathrm{pp}$.

4. R. E. BORCHERDS, Automorphic forms on $\mathrm{O}_{s+2,2}(R)$ and infinite products, Invent. Math. 120 (1995), no. 1, 161-213.

5. KATHRIN BRINGMANN and Ken ONO, The $f(q)$ mock theta function conjecture and partition ranks, Invent. Math. 165 (2006), no. 2, 243-266.

6. JAN HENDRIK BRUINIER and JENS FunKe, On two geometric theta lifts, Duke Math. J. 125 (2004), no. 1, 45-90.

7. JAN BRUINIER and KEN ONO, Heegner divisors, $L^{-}$ functions and harmonic weak Maass forms, Ann. of Math. (2) 172 (2010), no. 3, 2135-2181.

8. JAN HENDRIK BRUINIER and KEN ONO, Algebraic formulas for the coefficients of half-integral weight harmonic weak Maass forms, Adv. Math. 246 (2013), 198-219.

9. Y. ChOIE, M. KNOPP, and W. PRIBITKIN, Niebur integrals, mock automorphic forms and harmonic Maass forms: A retrospective on the work of the Rademacher school, preprint, 2014.

10. H. COHEN, $q$-identities for Maass waveforms, Invent. Math. 91 (1988), no. 3, 409-422.

11. W. Duke, Ö. ImAmoḠLU, and Á. Tóth, Rational period functions and cycle integrals, Abh. Math. Semin. Univ. Hambg. 80 (2010), no. 2, 255-264.

12. __ Cycle integrals of the $j$-function and mock modular forms, Ann. of Math. (2) 173 (2011), no. 2, 947-981.

13. __ Real quadratic analogs of traces of singular moduli, Int. Math. Res. Not. IMRN 2011, no. 13, 30823094.

14. _ , Regularized inner products of modular functions, to appear in Ramanujan Journal.

15. W. DUKE and Y. LI, Harmonic Maass forms of weight one, to appear in Duke Math. J.

16. STEPHAN EHLEN, On CM values of Borcherds products and weight one harmonic weak Maass forms, preprint.

17. AMANDA FOLSOM, What is... a mock modular form? Notices Amer. Math. Soc. 57 (2010), no. 11, 1441-1443.

18. BASIL GORDON, and RichaRd J. MCINTOSH, A survey of classical mock theta functions. Partitions, q-series, and Modular Forms, 95-144, Dev. Math., 23, Springer, New York, 2012.

19. G. H. HARDY and S. RAmANUJAN, Asymptotic formulae in combinatory analysis, Proc. London Math. Soc. (2) 17 (1918), 75-115, Collected Papers of Srinivasa Ramanujan, 276-309, AMS Chelsea Publ., Providence, RI, 2000.

20. DENNIS A. HEJHAL, Eigenvalues of the Laplacian for PSL(2,Z): Some new results and computational techniques, International Symposium in Memory of Hua Loo Keng, Vol. I (Beijing, 1988), 59-102, Springer, Berlin, 1991.

21. DEAN HiCKERSON, A proof of the mock theta conjectures, Invent. Math. 94 (1988), no. 3, 639-660. 


\section{Automorphisms and Equivalence Relations in Topological Dynamics}

David B. Ellis,

Beloit College, Wisconsin

Robert Ellis,

Beloit College, Wisconsin

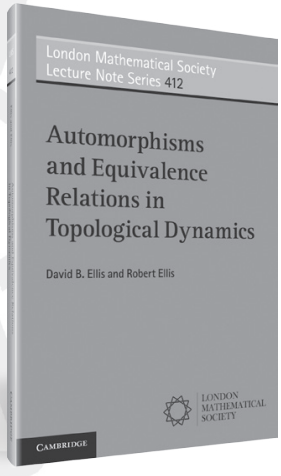

Focusing on the role that automorphisms and equivalence relations play in the algebraic theory of minimal sets provides an original treatment of some key aspects of abstract topological dynamics. Such an approach is presented in this lucid and self-contained book, leading to simpler proofs of classical results, as well as providing motivation for further study. This book is designed as both a guide for graduate students, and a source of interesting new ideas for researchers.

London Mathematical Society Lecture Note Series, No. 412

Paperback | 9781107633223 | June 2014 | $£ 45.00$

www.cambridge.org/lms412

\section{Optimal \\ Transportation}

Theory and Applications

Hervé Pajot,

Université de Grenoble

Yann Ollivier,

Université de Paris XI

Cedric Villani,

Université de Paris VI (Pierre et Marie Curie)

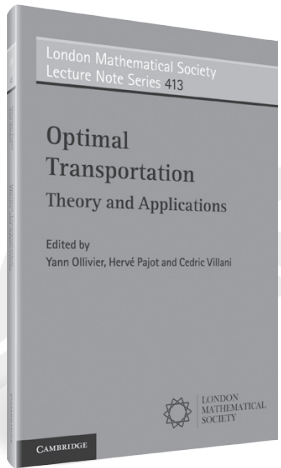

- Contains short courses which give an accessible introduction to problems of current interest, and research papers which present modern developments

- The book presents both the theory of optimal transport and some of its many applications

- Of interest to researchers in pure and applied mathematics, physics, computer science and economics

London Mathematical Society Lecture Note Series, No. 413 Paperback | 9781107689497 | July 2014 | £40.00

www.cambridge.org/lms413
22. _ On the seventh order mock theta functions, Invent. Math. 94 (1988), no. 3, 661-677.

23. F. HIRZEBRUCH and D. ZAGIER, Intersection numbers of curves on Hilbert modular surfaces and modular forms of Nebentypus, Invent. Math. 36 (1976), 57-113.

24. HenryK IWANIEC, Topics in Classical Automorphic Forms, Graduate Studies in Mathematics, 17, American Mathematical Society, Providence, RI, 1997, xii+259 pp.

25. HenRYK IWANIEC and EMmanuel Kowalski, Analytic Number Theory, American Mathematical Society Colloquium Publications, 53, American Mathematical Society, Providence, RI, 2004, xii+615 pp.

26. H. IWANIEC and P. SARNAK, Perspectives on the analytic theory of L-functions, GAFA 2000 (Tel Aviv, 1999). Geom. Funct. Anal. 2000, Special Volume, Part II, 705-741.

27. KEN ONO, Unearthing the visions of a master: Harmonic Maass forms and number theory, Current Developments in Mathematics, 2008, 347-454, Int. Press, Somerville, MA, 2009.

28. Soc. 57 (2010), no. 11, 1410-1419.

29. _ Algebraicity of harmonic Maass forms, Ramanujan J. 20 (2009), no. 3, 297-309.

30. HANS RADEMACHER, On the partition function $p(n)$, Proc. London Math. Soc. S2-43, no. 4, 241.

31. Atle SElberg, Über die Mock-Theta funktionen siebenter Ordnung, Arch. Math. Naturvidenskab 41 (1938); 3-15 in Collected Papers Vol. 1.

32. _ On the estimation of Fourier coefficients of modular forms, Proc. Sympos. Pure Math., Vol. VIII, pp. 1-15, Amer. Math. Soc., Providence, RI, 1965.

33. _ Reflections around the Ramanujan centenary, Ramanujan: Essays and Surveys, 203-213, Hist. Math. 22, Amer. Math. Soc., Providence, RI, 2001.

34. MARYNA VIAZOVSKA, CM values of higher Green's functions, preprint.

35. G. N. WAtson, The Final Problem: An account of the mock theta functions, J. London Math. Soc. S1-11, no. 1, 55, 1935.

36. Don ZAGier, Traces of singular moduli, Motives, Polylogarithms and Hodge Theory, Part I (Irvine, CA, 1998), 211-244, Int. Press Lect. Ser., 3, I, Int. Press, Somerville, MA, 2002.

37. , Ramanujan's mock theta functions and their applications (after Zwegers and Ono-Bringmann), Séminaire Bourbaki. Vol. 2007/2008. Astérisque No. 326 (2009), Exp. No. 986, vii-viii, 143-164 (2010).

38. _ Quantum modular forms, Quanta of Maths, 659-675, Clay Math. Proc., 11, Amer. Math. Soc., Providence, RI, 2010.

39. S. P. ZWEGERS, Mock $\theta$-functions and real analytic modular forms, a-series with applications to combinatorics, number theory, and physics (Urbana, IL, 2000), 269277, Contemp. Math., 291, Amer. Math. Soc., Providence, RI, 2001.

40. _ Mock Theta Functions, Utrecht Ph.D. thesis, (2002), ISBN 90-393-3155-3. 